\title{
Structural Variation in Genesis and Landfall Locations of North Atlantic Tropical Cyclones Related to SST
}

\author{
By Jonas Rumpf ${ }^{1 \star}$, Helga Weindl ${ }^{2}$, Eberhard Faust $^{2}$, and Volker Schmidt ${ }^{1}$ \\ ${ }^{1}$ Ulm University, Institute of Stochastics, 89069 Ulm, Germany; ${ }^{2}$ Munich Reinsurance Company, 80791 Munich, Germany
}

(Manuscript received March 13, 2009; Revised version received November 4, 2009)

\begin{abstract}
The influence of sea surface temperature on the locations of the genesis and of landfall of tropical cyclones in the North Atlantic is analyzed. For that purpose, these locations are calculated from HURDAT and split into two disjoint subsets according to whether sea surface temperature in the North Atlantic was above or below average in the year the corresponding storms occurred. Landfalls are investigated separately for the groups of cyclones categorized as tropical storms, minor hurricanes, or major hurricanes at the moment of landfall. The locations are considered realizations of inhomogeneous Poisson point processes, and the corresponding density functions are estimated with kernel estimation methods. In this way, any spatial structure inherent in the data is taken into account. These density functions are then compared with Monte Carlo methods from spatial statistics, which allows the detection of areas of statistically significant differences in the two sets with high and low SST, respectively. Results show many such areas, which is of relevance for the insurance industry and mathematical modelling of cyclones, as well as for decision support during the preparation for natural disasters.
\end{abstract}

\section{Introduction}

There is a strong connection between cyclone activity and sea surface temperature (SST) in the North Atlantic and other ocean basins, see, for example, Mann and Emanuel (2006), Holland and Webster (2007), and Saunders and Lea (2008). Gray (1979) also pointed out the necessity of a water temperature of $26.5^{\circ} \mathrm{C}$ to a depth of approximately $50 \mathrm{~m}$ for the formation of a tropical cyclone. For more details, see also Chan et al. (2001) and the references therein.

In recent years a vivid discussion has emerged on phases of low and high hurricane activity in the North Atlantic which is connected to a multidecadal swing of Atlantic SST. This multidecadal swing is thought to be basically an element of natural climate variability and called Atlantic Multidecadal Oscillation (AMO), see, for example, Goldenberg et al. (2001), Vellinga and $\mathrm{Wu}$ (2004), Knight et al. (2005), and Zhang and Delworth (2006). Recent research suggested that the AMO may influence hurricane activity through areal changes in the so-called Atlantic Warm Pool (AWP) which comprises warm surface waters of the Gulf of Mexico, the Caribbean Sea and the western tropical North Atlantic, see Wang et al. (2008). The reduction of the vertical wind shear in the Main Development Region (MDR) and the local enhancement of thermodynamic convective instability - both of which are preconditions of tropical cyclone formation and development - are coupled with a large AWP.

* Corresponding author.

e-mail: jonas.rumpf@uni-ulm.de
While it is already of scientific interest to investigate how the numbers of tropical cyclones vary with SST, there is also the question of variation in locations of cyclone genesis and cyclone landfall. More specifically, one can ask if changes in SST are accompanied by significant changes in the spatial distributions of the locations where cyclones form or those where they make landfall - regardless of the actual absolute numbers. In this report, we investigate which regions are subject to this kind of higher or lower relative frequencies of cyclone genesis or cyclone landfall due to SSTs which are above or below the long-term average.

While the aim of the investigation of Dailey et al. (2009) is similar to ours, we take a different approach at the problem: In a similar way as Dailey et al. (2009), we split the data into two disjoint subsets from a 'warm phase' and a 'cold phase'. Instead of then comparing the counts of points in certain subregions (a method similarly also suggested by Hall and Jewson (2008)), we estimate density functions for a point process model in the whole region of interest, i.e. the main development region of tropical cyclones and the complete (western) coastline of the North Atlantic. These density functions reflect the spatial structure inherent in the patterns formed by points of cyclone genesis or landfall points, respectively. Furthermore, they are standardized, and thus independent of the actual number of cyclones involved. Then, the standardized density functions are compared statistically with the help of Monte Carlo methods from spatial statistics to obtain regions of significant differences between the warm phase and the cold phase. 
Obvious applications of such an investigation include refined hazard and risk assessment in the insurance industry as well as implications for strategies in the preparation for and mitigation of natural disasters in the areas affected by tropical cyclones. Furthermore, mathematical and physical models of tropical cylone tracks, such as those suggested in Emanuel et al. (2006), Hall and Jewson (2007), and Rumpf et al. (2007; 2009), could be adjusted to better represent current SST conditions. In fact, the techniques we use for the estimation of the density functions are very similar to those that the aforementioned track simulation models use to estimate rates of cyclone genesis. Thus, the procedures we suggest for the comparison of subsets of historical data, should also be easily applicable to the comparison of these model outputs with the historical data or of the models with each other.

This paper is structured as follows: In Section 2, the data used for this study is discussed and definitions for the terms 'point of cyclone genesis' and 'point of landfall', as well as 'warm phase' and 'cold phase', which are used throughout the paper are given. The first part of Section 3 is dedicated to the explanation of the methods that are used to estimate density functions of the various point patterns under investigation, while Section 3.2 discusses the Monte Carlo methods for the comparison of these density functions. The results are all presented and interpreted in Section 4. A short summary and outlook in Section 5 conclude the paper.

\section{Data}

All cyclone track data used in this study is taken from the HURDAT best track data, see Jarvinen et al. (1984). For each tropical cyclone that occurred in the North Atlantic ocean basin between 1851 and 2007, this database contains (among other information not relevant for this study) the geographical position of the center of the cyclone and its wind speed, measured in intervals of 6 hours. The first registered position of a cyclone will be considered its 'point of genesis' for the purpose of this investigation. Note that there are some points of cyclone genesis in the data with a position over land, a situation that makes no meteorological sense, but is tolerated for lack of better data.

While each cyclone track in HURDAT by definition has exactly one point of genesis, it can have 0,1 or multiple points of landfall. For our investigation, a landfall occurs, when a point of measurement located over the ocean is followed by at least one point of measurement located over land. The location of the 'point of landfall' is then calculated as the midpoint of the line connecting these two points of measurement. While in reality, a landfall location is the exact point where a cyclone track crosses the coastline onto land, this interpolation technique will cause the points to lie slightly off the coastline, either on land or the ocean. However, the fact that the investigated points are not concentrated solely on the coast line makes them suitable for the mathematical investigation as a two-dimensional point pattern, see Section 3.1. Additionally, Figure 1 also shows that the imprecisions caused by this interpolation of the landfall points are fairly small.
The SST data used as the basis for the investigation was obtained from the Hadley Centre's sea ice and sea surface temperature data set HadISST1, see Rayner et al. (2003). Here, we use the SST anomalies from the months June through October of the years 1903-2005 relative to the mean in a region of the North Atlantic which is limited by $10^{\circ} \mathrm{N}$, $20^{\circ} \mathrm{N}, 80^{\circ} \mathrm{W}$, and $0^{\circ} \mathrm{E}$; this region roughly coincides with the MDR of North Atlantic tropical cyclones. Figure 2 shows this data in the form of the yearly deviations from the mean over the whole period; the solid line indicates a smoothed mean of the deviations. This smoothed mean clearly divides the observed period into four parts, two with an almost entirely positive smoothed mean, i.e. warmer than average temperatures, and two with cooler than average temperatures. The years with higher temperatures, running from 1926 to 1970 and from 1995 to 2005 , will be referred to as the 'warm phase' throughout this paper; they are marked in black in Figure 2. Marked in white are the years 1903-1925 and 1971-1994, which will be referred to as the 'cold phase'.

The use of the year 1903 as a starting point for our investigation is motivated by the fact that there is an apparent phase change in SST around the turn of the century in a detrended version of the time series. While it is difficult to determine the precise moment of this phase change, we feel that the slight uncertainty about this starting point does not influence our findings significantly. It is also worth noting that the reliability of HURDAT storm track data from earlier years is doubtful, especially with respect to the points of cyclone genesis. Equally, there is some measure of uncertainty involved with the HadISST1 SST data in the early years.

For those parts of our investigation that deal with landfall points, cyclones were categorized into three groups according to their intensity: In accordance with the Saffir-Simpson hurricane scale, cyclones with wind speeds of $63-118 \mathrm{~km} / \mathrm{h}$ are called 'tropical storms', those falling into Saffir-Simpson categories 1 and 2 (i.e. exhibiting wind speeds between 118 and $177 \mathrm{~km} / \mathrm{h}$ ) will be referred to as 'minor hurricanes', and all cyclones with wind speeds of more than $177 \mathrm{~km} / \mathrm{h}$ (i. e. Saffir-Simpson categories 3 - 5) are categorized as 'major hurricanes' for our investigation. Table 1 lists the numbers of landfall points from the investigated period where cyclones have made landfall while being categorized into one of the three mentioned groups. In brackets, the total numbers of cyclones are given whose highest wind speed over its life span fell into the corresponding group.

\section{$3 \quad$ Methodology}

\subsection{Density Estimation}

All point patterns considered in this paper are assumed to be realisations of inhomogeneous Poisson processes. This simple model for spatial point patterns assumes that the points of a pattern are placed independently of each other, an assumption that is justified by the nature of the investigated data: there is no indication that either starting points or points of landfall of tropical cyclones 'interact' in any way, especially not in data that was aggregated over multiple decades. For details on Poisson processes and their 
characteristics, see for example Diggle (2003), or Illian et al. (2008).

An inhomogeneous Poisson process is characterized by its so-called intensity function. To avoid confusion with 'hurricane intensity', we will refer to all intensity functions of Poisson processes as (standardized) density functions, since the concept of a Poisson intensity function is closely related to that of a (probability) density function.

Heuristically speaking, the value of the density function of a Poisson process at a certain location indicates the frequency of points occurring at that location. If the true density function is unknown - as is the case in all 'real' data - it has to be estimated from the data. Due to the limited flexibility of parametric models, which usually only allow a small number of parameters to be fitted to the data, we found non-parametric techniques, particularly variations of kernel density estimators, to be more suitable for our investigations. Kernel-based approaches - some of which bear similarities to the methods we employed - have already been used to estimate probability density functions of genesis locations of tropical cyclones, for example by Emanuel et al. (2006), Hall and Jewson (2007), and Rumpf et al. (2007; 2009). The specifics of these techniques will be discussed in the following.

The kernel estimator $\hat{\lambda}(x)$ of a density function $\lambda(x)$ at a location $x$ is defined as

$$
\hat{\lambda}(x)=\frac{1}{h^{2}} \sum_{i=1}^{n} K\left(d\left(x, X_{i}\right), h\right),
$$

where $X_{i}$ denotes the location of the $i$-th data point, $d(.,$. is the (spherical) Euclidean distance, $K$ the so-called kernel function, and $h>0$ the bandwidth. The kernel function can be interpreted as the basic shape of the probability mass that is assigned to each location where a data point occurred, while the bandwidth indicates the scale over which this mass is 'spread out'. Usually, kernel functions are chosen to be non-negative, symmetrical density functions. Frequently used kernel functions are the Epanechnikov kernel (see Epanechnikov (1969)), which in two-dimensional space is defined as

$$
K_{E}(x, h)=\left\{\begin{array}{cl}
\frac{2}{\pi}\left(1-\left(\frac{x}{h}\right)^{2}\right), & \text { if } 0<x<1, \\
0 & \text { else, }
\end{array}\right.
$$

and the standard normal kernel, given in $2 \mathrm{D}$ by

$$
K_{N}(x, h)=\frac{1}{2 \pi} \exp \left(-\frac{1}{2}\left(\frac{x}{h}\right)^{2}\right) .
$$

There appears to be consensus in the literature that the choice of bandwidth is much more relevant to the quality of the kernel estimator than the choice of kernel, see Diggle (2003), p.118, Illian et al. (2008), p.482, and Møller and Waagepetersen (2003), p.37. Due to the importance of finding a 'good' bandwidth, methods for objectively determining a bandwidth have been discussed in the literature. For example, the method of likelihood cross validation (LCV) determines the quality of a bandwidth by calculating log-likelihoods of each point of data from a density estimator that has been determined by leaving the respective point out. To be precise, the likelihood cross validation optimal bandwidth is given by the $h$ that maximises the function

$$
L C V(h)=\frac{1}{n} \sum_{i=1}^{n} \log \left(\hat{\lambda}_{-i}\left(X_{i}\right)\right),
$$

where $\hat{\lambda}_{-i}$ is the kernel estimator determined as in (1), with the sole change of leaving out the $i-$ th summand. Alternatively, the least squares cross validation (LSCV) approach is to find the $h$ that minimizes an approximation to the integrated squared difference between the density estimate and the true density. For details on these methods, see Silverman (1986).

Although it has an intuitive appeal, the kernel estimator has some disadvantages. For example, even when an automated method such as LCV is employed, the use of a single, fixed bandwidth in the whole observation window can lead to serious defects in the estimate: a large bandwidth that adequately smoothes the estimators in regions of low point density can easily wipe out most or all of the detail in regions with a higher density. Also, when using a kernel with bounded support (such as the Epanechnikov kernel), LCV will result in a bandwidth that is no less than the largest distance between a point and its closest neighbour in the considered data. This can lead to severe oversmoothing in data with outliers.

As a partial remedy for these problems, the generalized nearest neighbour (GNN) estimator $\tilde{\lambda}$ is defined in a similar way as the kernel estimator, but with a bandwidth that is location-dependent:

$$
\tilde{\lambda}(x)=\frac{1}{r_{k}(x)^{2}} \sum_{i=1}^{n} K\left(d\left(x, X_{i}\right), r_{k}(x)\right),
$$

where $r_{k}(x)$ is the distance of the location $x$ from the $k$-th nearest data point and $0<k<n$. Through its variable bandwidth, the GNN estimator smoothes less in regions of high point density and more in regions of fewer points. The definition of $r_{k}(x)$ can, in the case of outliers, lead to very large bandwidths. Still, it respects regional variations in point density better, since large bandwidths do not only include points far away from $x$ into the estimate at $x$, but at the same time they shrink the estimate at $x$ by 'stretching out the kernel' for all observations regarded at $x$. Details on the GNN estimator can be found in the book by Silverman (1986), who also suggests the choice of $k=[\sqrt{n}]$. While in principle, an optimal $k$ could also be found by likelihood cross validation, an actual optimization of this parameter is computationally too expensive for many practical applications. A comparison of the likelihood scores (calculated as in (4) with $k$ as the variable instead of $h$ ) for $k=n^{\frac{1}{3}}$ and $k=n^{\frac{2}{3}}$ has shown only small differences with a slight advantage for $k=[\sqrt{n}]=n^{\frac{1}{2}}$.

\subsection{Monte Carlo Tests}

The main goal of this investigation is the statistical comparison of two point patterns - one from the warm phase and one from the cold phase. The pairs of hypotheses to be tested are of the form

$$
H_{0}: \lambda=\mu \text { vs. } H_{1}: \lambda \neq \mu,
$$


where $\lambda$ and $\mu$ are the two density functions underlying the two sets of data, which are considered realizations of inhomogeneous Poisson processes on some observation window $W$, compare Section 3.1. Additionally, when instead of the global hypotheses of (6), the equality of the two density functions at a specific location $x_{0}$ is of interest, the hypotheses

$$
H_{0}: \lambda\left(x_{0}\right)=\mu\left(x_{0}\right) \text { vs. } H_{1}: \lambda\left(x_{0}\right) \neq \mu\left(x_{0}\right)
$$

are tested. Since the statistical distributions involved in testing these hypotheses are not analytically known, and no distributional assumptions (such as normality or similar standard assumptions) are obvious, we use the Monte Carlo methods suggested by Kelsall and Diggle (1995): Let $x_{1}, \ldots, x_{n_{1}}$ and $y_{1}, \ldots, y_{n_{2}}$ denote the two sets of data points and $\lambda$ and $\mu$ their underlying density functions, respectively. To test the global hypotheses (6), the following method can be employed:

1. Calculate estimates $\hat{\lambda}$ and $\hat{\mu}$ from $x_{1}, \ldots, x_{n_{1}}$ and $y_{1}, \ldots, y_{n_{2}}$.

2. Calculate the log-ratio of these estimates $\hat{r}_{0}=\log \frac{\hat{\lambda}}{\hat{\mu}}$.

3. Randomly reassign $x_{1}, \ldots, x_{n_{1}}$ and $y_{1}, \ldots, y_{n_{2}}$ into two groups of sizes $n_{1}$ and $n_{2}$.

4. Calculate new estimates $\hat{r}_{i}=\log \frac{\hat{\lambda}_{i}}{\hat{\mu}_{i}}$ from the data reassigned in step 3 .

5. Repeat steps 3 and 4 for $i=1, \ldots, m$, with $m$ sufficiently large.

6. Calculate the integrals $s_{i}=\int_{W} \hat{r}_{i}^{2}(x) d x$ for $i=0, \ldots, m$.

7. The $p$-value of the test is then $p=\frac{k+1}{m+1}$, where $k=$ $\#\left\{j: s_{j}>s_{0}\right\}$.

The testing procedure described above relies on two basic facts: Firstly, note that $H_{0}$ in this case can be rephrased as "the separation of the data in two groups is random". Thus, one can easily see that the estimates $\hat{r}_{i}$ and consequently also the integrals $s_{i}$ have been obtained under the assumption of $H_{0}$. Furthermore, it is clear that under $H_{0}$, the log-ratio $r=\log \frac{\lambda}{\mu}$ is equal to zero. Thus, a large value of $s_{0}$ indicates a large deviation from $H_{0}$. The question what 'large' means in this context is then answered in step 7 by comparing $s_{0}$ with $s_{i}, i=1, \ldots, m$, which represent a sample from the distribution underlying the $s_{i}$ under $H_{0}$. Besides the $s_{i}$, various other statistics, such as the Kullback-Leibler divergence, could be employed to measure the deviations between $\lambda$ and $\mu$. For reasons of conciseness, we restrict ourselves here to the above definition of the $s_{i}$.

Note that the density estimates calculated in step 4 above are heavily influenced by the group sizes $n_{1}$ and $n_{2}$, so group sizes that differ strongly will always result in $H_{0}$ being rejected, even if the structures of the two density estimates are very similar. To avoid this and to compare the conditional densities of the two data sets, one can standardize the density estimates from step 4 by dividing them by the sizes of the respective data set. This is done for all data considered in this paper, since the data sets from the cold and the warm phase both differ greatly in size, as can be seen from Table 1.

To test the hypotheses of (7), a procedure analogous to the one described above can be used:
1. Calculate $\hat{r}_{0}\left(x_{0}\right), \hat{r}_{1}\left(x_{0}\right), \ldots, \hat{r}_{m}\left(x_{0}\right)$ as described above.

2. Calculate $p\left(x_{0}\right)=\frac{\#\left\{j: \hat{r}_{j}\left(x_{0}\right)<\hat{r}_{0}\left(x_{0}\right)\right\}}{m+1}$.

3. If $p\left(x_{0}\right)<\alpha / 2$ or $p\left(x_{0}\right)>1-\alpha / 2$, then $\lambda\left(x_{0}\right)$ is considered significantly different from $\mu\left(x_{0}\right)$ at a level of significance $\alpha$, i. e. $H_{0}$ is rejected.

This procedure for the local hypotheses simply applies the same ideas used to test the global hypotheses, it just replaces the integrals $s_{i}$, which aggregate the values of $\hat{r}_{i}$ over the whole observation window, by the value of $\hat{r}_{i}$ at a particular location of interest $x_{0}$.

For more details on the Monte Carlo testing procedures described in this section, see Kelsall and Diggle (1995) and the references therein. Also, in Illian et al. (2008), p.455ff, Monte Carlo tests are described more extensively in a point process context.

\section{Results}

In this section, the results of the comparison between data sets from the two different temperature phases are presented. Recall that, for the purpose of these comparisons, the investigated point patterns are assumed to be realizations of inhomogeneous Poisson processes, the density functions of which are estimated with a generalized nearest neighbour estimator as well as with a kernel estimator of type (1). For the kernel estimators, the standard normal kernel is used with a bandwidth determined by likelihood-cross-validation, while for the generalized nearest neighbour method, the Epanechnikov kernel is used and the parameter $k$ is chosen to be $k=[\sqrt{n}]$ (see Section 3.1). The optimal bandwidths for the kernel estimators of type (1) are given in Table 2. In all figures discussed in this section, red colours mark the highest values, intermediate values are marked green, and deep blue indicates the lowest values of the illustrated characteristic.

Note that for all investigations described in this section, the data is split into two disjoint subsets: one from the warm phase and one from the cold phase. The purpose of this is to detect potentially significant differences in the structures of the data sets between these two temperature phases. Additionally, one could also investigate structural differences between the complete data from both temperature phases and a subset of it, e.g. the data from the warm phase. However, the problem with this kind of investigation is that since the warm phase data is a subset of the complete data, there is a strong influence of the warm phase data on the estimated density function of the complete data. In other words: the structure of the estimated density function of the complete data is predictably much more similar to that of the warm phase data than to that of the cold phase data, simply because there is more data from the warm phase. Thus, not much insight would be gained in comparing the complete data with a subset of it and we restrict our investigations to the comparison of cold phase and warm phase data.

\subsection{Points of Cyclone Genesis}

The first pair of point patterns to be compared are the points of cyclone genesis from the warm phase and the cold 
phase, as they have been defined in Section 2. Figures 3 and 4 show the density estimates resulting from the GNN method together with the corresponding points of cyclone genesis from the warm phase and the cold phase, respectively. In Figure 5, the log-ratio of the two estimates is shown. The analogous results obtained with a kernel estimate of type (1) look very similar and are thus not depicted separately.

The black points in Figures 3 and 4 mark the locations of the 592 and 363 historical points of cyclone genesis contained in the warm phase data and the cold phase data, respectively (cf. also Table 1). These numbers can easily be considered large enough for stable density estimates. However, if one were interested in smaller subsets of the data, say, from certain shorter time periods, the issue of data sparsity would have to be looked at more closely. If the resulting density estimates then were considered too unstable for a meaningful interpretation, one might think of departing from estimating density functions from the historical data completely and looking at genesis potential indices (GPI). This type of index, discussed for example in Emanuel and Nolan (2004) and Camargo et al. (2007), uses information about environmental factors such as low-level vorticity or vertical wind shear to calculate probabilities of cyclone genesis in regions of interest. While this would resolve the issue of the amount of cyclone genesis points available, at the same time it would raise the issue of the reliability of the data needed for the GPI. Consequently, we feel that for statistical analyses of point patterns, it is ultimately better to rely on the actual point patterns, as long as they are reasonably large.

Note that all estimators in Section 4 have been standardized by the number of points in the respective data set, because otherwise all structural differences would be obscured by the fact that there are over $60 \%$ more points in the warm phase data than in the cold phase data. Thus, areas marked red in Figure 5 are not caused by a larger absolute number of points occurring in that area during a warm phase. Rather, large values of the log-ratio are indicative of the conditional probability that a point occurs in that area, under the condition that a point actually does occur, being higher during the warm phase than during the cold phase. An analogous explanation holds for low values of the log-ratio.

In a next step it is of interest which values of the log-ratio of standardized density estimates are actually significant, i.e. where a significant difference between warm phase and cold phase occurs. To answer this question, the pair of local hypotheses (7) was tested at a level of significance $\alpha=0.05$ with the procedure described in Section 3.2 for all pixels located over sea in Figure 5. Areas of significantly large values have been overlaid with a black grid, and areas of significantly low values are marked by a white grid. Again, the results obtained using kernel estimation of type (1) are not depicted in a separate image, since they are visually almost indistinguishable from those obtained using the GNN estimator. Table 3 lists the percentages of the observation area where the null hypothesis (7) of equal density values is rejected. Additionally, the results of the Monte Carlo tests of the global hypotheses (6) of overall equal density functions are shown in Table 4 . All tests were performed with $m=999$.
In Figures 3 through 5, some structural differences between the warm phase and the cold phase data are clearly visible. First and foremost, the two significant maxima of the logratio of standardized density functions around the Yucatan peninsula indicate that these two areas appear to be much more prone to the formation of tropical cyclones during the warm phase than during the cold phase - relatively speaking, i. e. as opposed to other regions of the observation window. A similar observation can be made for areas east and southeast of Puerto Rico, and again, to a lesser extent, around $40^{\circ} \mathrm{W}$ and $20^{\circ} \mathrm{N}$, and off the west African coast (cf. Figure 5). Additionally, the areas with a significantly higher density during the cold phase appear to be concentrated on the northern and southern edges of the observation window. However, one has to be careful when interpreting these areas, since some of them cover areas which contain virtually no points in either of the two data sets. This suggests that some of those differences might be caused by the fact that the bandwidth for the estimators (see Section 3.1) tends to be higher for the cold phase data due to the smaller number of points in that set of data (compare Tables 1 and 2). Still, this is certainly not the case for a large area northeast of the Bahamas, where the standardized density of cyclone genesis is clearly much higher during the cold than during the warm phase, again in contrast to other parts of the observation window.

When interpreting these results meteorologically, it is important to remember that the results described above are obtained with standardized density functions, i. e. they do not depend on the absolut number of cyclones occurring in each phase, but rather on relative probabilities of cyclones occurring in a certain area during a certain phase. It is also worth restating that the choice of estimation method does not appear to make a large difference with respect to these results, since the areas of significant differences between warm phase and cold phase appear to have the same size and structure when employing GNN estimation or kernel estimation of type (1).

The comparatively lesser density of genesis points east and northeast of Florida and the Bahamas during warm phase years might be - among other factors - due to an enhanced vertical wind shear during warm phase years in this region. Enhanced vertical wind shear tends to suppress tropical cyclone activity. The increased vertical wind shear in the region east and northeast of Florida and the Bahamas during the AMO warm phase has been demonstrated on the basis of reanalysis data, see Figure 11a of Wang et al. (2008).

Kossin and Vimont (2007) provide further data that helps to interpret our results. They describe the so-called Atlantic Meridional Mode (AMM), which represents the leading mode of basin-wide coupled ocean-atmosphere interaction between SST and low-level winds. According to these authors, the AMO is an important mechanism to excite the AMM. The composite figure that they provide for the 5 strongest AMM years (Figure 3 of Kossin and Vimont (2007)), which also represent warm phase years according to the AMO, show a pattern where the bulk of genesis points is concentrated in the MDR east of the Caribbean islands. This pattern is due to anomalously warm waters and anomalously reduced 
vertical wind shear in this area as opposed to areas farther north. In those years, we also have anomalously warm waters in the western Gulf of Mexico and farther south along the eastern coast of Central America, together with reduced vertical shear in this area (see Figure 3 of Kossin and Vimont (2007)). This might explain that we also find a significantly higher (relative) genesis density during the warm phase in this region (see Figure 5). In addition, the composite for the 5 weakest AMM years (Figure 3 of Kossin and Vimont (2007)), which coincide with AMO negative years (i. e. cold phase years), shows enhanced wind shear over the MDR extending far to the west and also cool SST anomalies in particular in the central and easten parts of the MDR. This can be associated with a significantly reduced density of genesis locations in this area during the cold phase, which can be found in Figure 5. Furthermore, the relatively more favourable genesis conditions in the subtropics west and east of Florida and the south eastern US coastline seem to be fostered by the reduced vertical wind shear and almost no anomalous reduction in SST which Kossin and Vimont (2007) find in that area.

\subsection{Points of Cyclone Landfall}

In this section, the points of cyclone landfall, as they were defined in Section 2, are investigated. As mentioned, the landfall points are split into three different classes according to the wind speed at the point of landfall; i. e., in addition to the complete set of landfall points, we investigate cyclones that are tropical storms, minor hurricanes, or major hurricanes at landfall separately. Apart from this, the investigations are completely analogous to those of Section 4.1. The observation window for the landfall points consists of the coastline of the North Atlantic, dilated by 8 pixels into all directions, and limited by $8.5^{\circ} \mathrm{N}, 50^{\circ} \mathrm{N}, 100^{\circ} \mathrm{W}$, and $50^{\circ} \mathrm{W}$. The very small number of landfall points not contained in this strip along the coast are considered artefacts of the approximation method used to obtain the landfall points (cf. Section 2) and are thus ignored for this investigation. Note again that for the reasons given in Section 4.1, all density estimators have been standardized by the number of points in the respective data sets, i. e. the number of landfalls in the respective phases. As in Section 4.1, all tests were performed with $m=999$.

For obvious reasons, the number of points in the different investigated subsets of landfall points is smaller than in the set of genesis points, cf. Table 1 . This makes the density estimates somewhat less reliable. However, since the observation window also is much smaller, the estimates and their comparisons still have meaningful interpretations, as can be seen from the results of a simulation study assessing the mean integrated squared error (MISE) of the density estimation on the observation window $W$ used for the points of cyclone landfall. In this simulation study, we simulated 1000 realizations of an inhomogeneous Poisson process with $k$ expected points (for $k$ between 10 and 600) in $W$ with the density $\hat{f}$ that had been estimated from the 66 points of major hurricane landfall. From these simulated point patterns, we re-calculated (standardized) density estimates $\hat{g}_{i}^{(k)}, i=1, \ldots, 1000$ using the GNN estimator and then calculated an approximation to the mean integrated squared error, i. e. the following index of deviation between the "true" and the re-estimated density:

$$
\Delta^{(k)}=\frac{1}{1000} \sum_{i=1}^{1000} \Delta_{i}^{(k)}=\frac{1}{1000} \sum_{i=1}^{1000} \int_{W}\left(\hat{g}_{i}^{(k)}(x)-\hat{f}(x)\right)^{2} d x
$$

The MISE is one of the most commonly used measures of error in kernel density estimation, see, for example Wand and Jones (1995), p.94ff., and Silverman (1986), p.35f. The resulting values of $\Delta^{(k)}$ are given as a function of $k$ in Figure 12. This figure shows that while the values clearly decline monotonously with an increasing number of expected points, the MISE - and therefore the density estimate - can be considered stable from around 40 points. Thus, while the MISE for 66 points (the size of the smallest point pattern) is approx. twice as large as for 518 points (the size of the largest point pattern), we can consider all our estimates stable enough with respect to MISE. Furthermore, when considering the influence of the amount of data on the results of this study, note that less points in the original point patterns also means less points in the randomly reassigned "simulated point patterns" (see Section 3.2). Thus, any lower reliability in densities estimated from smaller point patterns is reflected in the densities estimated from the corresponding "simulated point patterns". Consequently, potential data sparseness does not directly influence significance results of the Monte Carlo tests, and therefore can be considered of secondary importance to the results presented in this manuscript.

If the data were even more sparse, one might consider simulating cyclone tracks with models such as those from Emanuel et al. (2006), Hall and Jewson (2007), and Rumpf et al. $(2007 ; 2009)$. In this way, one could create large amounts of data and from this, a larger number of landfall points than in the original data. However, this would raise the question of how well any simulation models are able to reproduce the distributions of the landfall statistics - or any other aspect of the historical data. All of these models are at least in part stochastic and certainly quite complex, which makes model checking an important and non-trivial issue that, to our knowledge, has not yet been resolved, see also e. g. Hall and Jewson (2008). Therefore, as long as there is no clear result that a simulation model correctly reproduces the desired landfall distributions, we feel that to investigate characteristics of historical data, it is best to rely simply on the historical data itself, if possible, and not on simulations that introduce new uncertainties.

Figure 6 shows the log-ratio of the standardized estimated density fields of points of landfall of all categories, obtained with a GNN estimator. In Figures 7, 8, and 9, the corresponding values are plotted for the subsets of points of landfall of cyclones categorized as tropical storms, minor hurricanes, and major hurricanes, respectively. As explained in Section 4.1 , areas overlaid with a white grid are those where the value of the standardized density estimate is significantly larger during the cold phase than during the warm phase, and the black grid points indicate areas where the same is the case for the warm phase. In Tables 5 and 6 , the percentages of the observation area where the null hypothesis of equal standardized density values (see (7)) is rejected are given for the use of the GNN estimator and the kernel estimator of type 
(1), respectively. Finally, the results of the Monte Carlo tests of the global hypotheses (6) of overall equal standardized density functions are shown in Table 7 .

Figures 6 through 9 show many areas with significant differences in the estimated density functions (using GNN estimation) of landfall points between the cold and the warm phase. The results obtained with the GNN estimator and the kernel estimator of type (1) are not quite as consistent as they were for the points of cyclone genesis (cf. Section 4.1), but still similar. The increased variability can largely be attributed to the smaller observation window and the fact that the different classes contain a much smaller number of points (cf. Table 1). Still, the common threads are dominant, and thus, the results of the kernel estimation of type (1) are not depicted separately. For tropical storms, the standardized landfall density around southern Florida, along the US east coast between Maryland and Massachusetts, and from eastern Cuba to Haiti is significantly lower during the warm phase than during the cold phase. The opposite is the case around New Orleans and the Mexican state of Veracruz. When considering only minor hurricanes, a significantly higher standardized landfall density during the warm phase is detected for most of Florida, while around New Orleans the standardized landfall density is again higher during the cold phase. There are large areas with significant differences in the estimated standardized density functions for the points of landfall of major hurricanes. However, the different estimators show some inconsistencies, but they agree on the fact that in southeastern Florida, the standardized density appears to be significantly higher during the warm phase. Finally, Table 7 shows that the global hypothesis of equal standardized density functions can not be rejected in most cases, although the percentages of areas with significant differences tend to be higher than in the investigation of genesis points (cf. Tables 3, 5, and 6). This indicates that the differences between the warm phase and the cold phase are not very large and rather localized in nature.

Regarding a meteorological explanation for relatively enhanced landfall frequencies of minor and major hurricanes around southern Florida and - in the case of major hurricanes - at even lower latitudes in Cuba (compared with other parts of the observation window; see Figures 8 and 9), one has to start from the following findings: During warm phase years, the bulk of genesis locations is shifted equatorward, and in particular a substantial number of storms form in the central part of the tropical Atlantic (see Section 4.1 and Figure 5). Combined with typical subtropical steering flow in this region, the corridor which such southern storms have to pass through is very conducive to intensification, at least in the Caribbean Sea. This combination of typical steering flow and conditions prone to intensification is consistent with a positive signal of hurricanes hitting Florida and in the case of major hurricanes hitting areas even further to the south. The argument of Kossin and Vimont (2007) that the equator-ward amd eastward shift in genesis region causes storms to reach higher intensities at landfall also fits in well with this observation.

\section{Summary and Outlook}

In this paper, we investigated the statistical effects of SST on the structures of the areas of cyclone genesis and cyclone landfall. For this purpose we used two kernel estimation techniques to estimate the density functions of various point patterns obtained from subsets of HURDAT and then compared these density functions using Monte Carlo methods. These techniques account for the spatial structure of the investigated point patterns. In this way, we detected areas of significant differences in relative densities of genesis and landfall points, independently of the absolute numbers of cyclone genesis or cyclone landfall. For the points of cyclone genesis, the main results did not depend on the estimation method used, while for the landfall points, there were some differences. These were mainly due to the smaller observation window and smaller number of points in the investigation of landfall points.

The main focus of our investigation was the detection of structural differences in the various density estimates that were independent of the actual number of points contained in the respective data sets. It is of course valid to ask what the results would be if they were calculated from non-standardized density functions, i. e. if they considered the 'first-order effect' of (warmer) SST: improved conditions for (increased) TC activity. Recalculating all results without standardizing the density estimates by the number of points in the respective point patterns - but accounting for the fact that there are more years in the warm phase (56) than in the cold phase (47) - we get the expected results which are displayed in Figures 10 and 11: Almost all structural information is obscured by the fact that points are more frequent overall during the warm phase than during the cold phase (see Table 1). Figure 10 shows the non-standardized GNN density estimator for points of tropical cyclone genesis during the cold phase with a colour scale identical to that of Figure 3. Comparing Figures 3 and 10, one can easily see that the density is higher during the warm phase than during the cold phase almost everywhere. Anlogous results are obtained for the points of cyclone landfall. For the GNN estimator, the areas where this effect is statistically significant take up between $76.9 \%$ and $89.0 \%$ of the observation window (kernel estimator of type (1): $52.2 \%$ to $66.6 \%$ ) for the various point patterns, and there are no areas at all with a significantly higher density during the cold phase in any of the point patterns investigated; compare with Tables 3,5 , and 6 . The regions where the hypothesis of equal values of the estimated non-standardized densities can not be rejected are basically those that are marked in turqoise and deeper blues in Figures 5 through 9. This is exemplified for the points of cyclone genesis in the comparison of Figure 5 (standardized densities) with Figure 11 (non-standardized densities). Moreover, the hypothesis of overall equal intensity fields is rejected for all non-standardized point patterns with $p$-values of no more than 0.02; compare with Tables 4 and 7 .

It is clear that Atlantic SST alone is not the only factor determining cyclone activity in the North Atlantic. Recent research has shown that, for example, the difference between Atlantic SST and Indo-Pacific SST influences vertical wind shear over the MDR, which in turn has an effect on tropical 
cyclones, see Latif et al. (2007) and Swanson (2008). Also, as mentioned in Section 1, thermodynamic convective instability is another important precondition for the development of tropical cyclones. Consequently, it might be of interest to investigate subsets of HURDAT obtained according to more sophisticated criteria: instead of using simply the smoothed yearly deviations of Atlantic SST from the mean, one could base the separation of the data on information about vertical wind shear, the difference in SST between the tropical Atlantic and the Indo-Pacific tropical domain, ENSO, or any combination of other climatological criteria. Note that when doing so, one carefully has to consider the actual intended application when choosing which criteria to use. Some of these climatological phenomena can only be forecast a few months in advance, which would probably not be very helpful for the assessment of insurance risks. Still, the Monte Carlo methods used in our investigation would in any case be applicable to any such subsets, since they are only based on point patterns.

From a different perspective, one could also use these methods to compare the output of simulation models for tropical cyclone tracks (see Emanuel et al. (2006), Hall and Jewson (2007), and Rumpf et al. (2007; 2009)) with the historical data. This comparison could then serve as a tool for model evaluation.

Furthermore, other methods for density estimation than those used here (see Section 3.1) can also be employed, see for example Silverman (1986), or Scott (1992). Finally, for large enough sets of data, one could also abandon Monte Carlo methods and instead apply limit theorems for kernel estimators (see, for example, Cacoullous (1966)) to obtain asymptotic tests of the hypothesis of equal densities.

\section{Acknowledgments}

The authors would like to thank Genia Malinkowa for her excellent contributions to the programming work and the data analysis that form the basis of the results presented in this paper. We are also grateful to two anonymous reviewers whose constructive comments helped to improve the manuscript.

\section{REFERENCES}

Cacoullous, T., 1966. Estimation of a multivariate density. Ann. I. Stat. Math. 18 (2), 179-189.

Camargo, S.J., Emanuel, K.A., and Sobel, A.H., 2007. Use of a genesis potential index to diagnose ENSO effects on tropical cyclone genesis. J. Climate 20, 4819-4834.

Chan, J., Duan, Y., and Shay, L., 2001. Tropical cyclone intensity change from a simple ocean-atmosphere coupled model. $J$. Atmos. Sci. 58 (2), 154-172.

Dailey, P.S., Zuba, A., Ljung, G., Dima, I.M., and Guin, J., 2009. On the relationship between North Atlantic sea surface temperatures and U.S. hurricane landfall risk. J. Appl. Meteorol. Clim. 48 (1), 111-129.

Diggle, P.J., 2003. Statistical Analysis of Spatial Point Patterns, 2nd Edition., Arnold, London
Emanuel, K.A., and Nolan, D.S., 2004. Tropical cyclone activity and global climate. Proc. of the 26th Conference on Hurricanes and Tropical Meteorology, Miami, FL, 240-241.

Emanuel, K., Ravela, S., Vivant, E., and Risi, C., 2006. A statistical deterministic approach to hurricane risk assessment. B. Am. Meteorol. Soc. 87, 299-314.

Epanechnikov, V.A., 1969. Non-parametric estimation of a multivariate probability density. Theor. Probab. Appl. 14 (1), $153-158$.

Goldenberg, S.B., Landsea, C.W., Mestas-Nuñez, A.M. and Gray, W.M. 2001. The recent increase in Atlantic hurricane activity: causes and implications. Science 293, 474-479.

Gray, W.M., 1979. Hurricanes: Their formation, structure and likely role in the tropical circulation. In: Meteorology Over Tropical Oceans, (ed. D.B. Shaw), Roy. Meteor. Soc., Berkshire, $155-218$.

Hall, T.M., and Jewson, S., 2007. Statistical modeling of North Atlantic tropical cyclone tracks. Tellus 59A, 486-498.

Hall, T.M. and Jewson, S., 2008. Comparison of local and basinwide methods for risk assessment of tropical cyclone landfall. J. Appl. Meteorol. Clim. 47, 361-367.

Holland, G.J., and Webster, P.J., 2007. Heightened tropical cyclone activity in the North Atlantic: natural variability or climate trend? Philos. Trans. R. Soc. London, Ser. A 365 (1860), 2695-2716.

Illian, J., Penttinen, A., Stoyan, H., and Stoyan, D., 2008. Statistical Analysis and Modelling of Spatial Point Patterns, J. Wiley \& Sons, Chichester

Jarvinen, B.R., Neumann, C.J., and Davis, M.A.S., 1984. A tropical cyclone data tape for the North Atlantic basin, 1886-1983, contents, limitations and uses. NOAA Tech. Memo. NWS NHC 22, Miami, FL

Kelsall, J.E., and Diggle, P.J., 2001. Non-parametric estimation of spatial variation in relative risk. Stat. Med. 14, 2335-2342.

Knight, J.R., Allan, R.J., Folland, C.K., Vellinga, M., and Mann, M.E., 2005. A signature of persistent natural thermohaline circulation cycles in observed climate. Geophys. Res. Lett. 32 L20708

Kossin, J.P., and Vimont, D.J., 2007. A more general framework for understanding atlantic hurricane variability and trends. B. Am. Meteorol. Soc. 88 (11), 1767-1781.

Latif, M., Keenlyside, N., and Bader, J., 2007. Tropical sea surface temperature, vertical wind shear, and hurricane development. Geophys. Res. Letters 34, L01710

Mann, M.E., and Emanuel, K.A., 2006. Atlantic hurricane trends linked to climate change. EOS, Transactions, American Geophysical Union 87, 233-244.

Møller, J., and Waagepetersen, R.P., 2003. Statistical Inference and Simulation for Spatial Point Processes, Chapman \& Hall, New York

Rayner, N.A., Parker, D.E., Horton, E.B., Folland, C.K., Alexander, L.V., Rowell, D.P., Kent, E.C., and Kaplan, A., 2003. Global analyses of sea surface temperature, sea ice, and night marine air temperature since the late nineteenth century. $J$. Geophys. Res. 108 (D14) 4407

Rumpf, J., Weindl, H., Höppe, P., Rauch, E., and Schmidt, V., 2007. Stochastic modelling of tropical cyclone tracks. Math. Meth. Oper. Res. 66 (3), 475-490.

Rumpf, J., Weindl, H., Höppe, P., Rauch, E., and Schmidt, V., 2009. Tropical cyclone hazard assessment using model-based track simulation. Nat. Hazards 48 (3), 383-398

Saunders, M.A., and Lea, A.S., 2008. Large contribution of sea surface warming to recent increase in Atlantic hurricane activity. Nature 451 (7178), 557-560.

Scott, D.W., 1992. Multivariate Density Estimation: Theory, Practice, and Visualization, Wiley, New York

Silverman, B.W., 1986. Density Estimation for Statistics and Data Analysis, Chapman \& Hall, London 
Swanson, K.L., 2008. Nonlocality of Atlantic tropical cyclone intensities. Geochem. Geophys. Geosyst. 9, Q04V01.

Vellinga, M., and Wu, P., 2004. Low-latitude freshwater influence on centennial variability of the Atlantic thermohaline circulation. J. Clim. 17, 4498-4511.

Wand, M.P., and Jones, M.C., 1995. Kernel Smoothing, Chapman \& Hall, London

Wang, C., Lee, S.-K., and Enfield, D.B., 2008. Atlantic warm pool acting as a link between Atlantic multidecadal oscillation and Atlantic tropical cyclone activity. Geochem. Geophys. Geosyst. 9, Q05V03.

Zhang, R., and Delworth, T.L., 2006. Impact of Atlantic multidecadal oscillations on India/Sahel rainfall and Atlantic hurricanes. Geophys. Res. Lett. 33, L17712. 


\section{Figure legends}

Figure 1. Investigated points of tropical cyclone landfall along the North Atlantic ocean basin

Figure 2. North Atlantic SST data; columns: deviations from the mean (in degree Celsius); solid line: smoothed mean of deviations; years marked white: cold phase; years marked black: warm phase

Figure 3. Standardized GNN density estimator for points of cyclone genesis from the warm phase. Historical cyclone genesis sites are marked by black points.

Figure 4. Standardized GNN density estimator for points of cyclone genesis from the cold phase. Historical cyclone genesis sites are marked by black points.

Figure 5. Log-ratio of standardized GNN density estimators for points of cyclone genesis from the warm phase and the cold phase and areas of statistically significant differences at level of significance $\alpha=0.05$. Areas overlaid with a black grid have a significantly higher standardized density during the warm phase, areas overlaid with a white grid have a significantly higher standardized density during the cold phase.

Figure 6. Log-ratio of standardized GNN density estimators for points of cyclone landfall (all categories) from the warm phase and the cold phase and areas of statistically significant differences at level of significance $\alpha=0.05$. Meaning of black and white grids as in Figure 5.

Figure 7. Log-ratio of standardized GNN density estimators for points of cyclone landfall (tropical storms) from the warm phase and the cold phase and areas of statistically significant differences at level of significance $\alpha=0.05$. Meaning of black and white grids as in Figure 5.

Figure 8. Log-ratio of standardized GNN density estimators for points of cyclone landfall (minor hurricanes) from the warm phase and the cold phase and areas of statistically significant differences at level of significance $\alpha=0.05$. Meaning of black and white grids as in Figure 5 .

Figure 9. Log-ratio of standardized GNN density estimators for points of cyclone landfall (major hurricanes) from the warm phase and the cold phase and areas of statistically significant differences at level of significance $\alpha=0.05$. Meaning of black and white grids as in Figure 5 .

Figure 10. Non-standardized GNN density estimator for points of cyclone genesis from the cold phase. Historical cyclone genesis sites are marked by black points. Colour scale identical to that of Figure 3.

Figure 11. Log-ratio of non-standardized GNN density estimators for points of cyclone genesis from the warm phase and the cold phase and areas of statistically significant differences at level of significance $\alpha=0.05$. Areas overlaid with a black grid have a significantly higher non-standardized density during the warm phase. Note that the colours are the same as in Figure 5, although the numerical values are different, since the values depicted in Figure 5 can be obtained from those of this figure by multiplication with a constant scaling factor, i. e. the ratio of the standardization constants of the two densities.

Figure 12. Results of a simulation study assessing the mean integrated squared error (MISE) of a density estimate on the observation window $W$ used for the points of cyclone landfall. Horizontal axis: expected number of points in the point pattern; vertical axis: (approximate) MISE of the kernel density estimate. 
Table 1. Numbers of landfalls (cyclones) in the different groups and phases

\begin{tabular}{cccc}
\hline category & $1903-2005$ & warm phase & cold phase \\
\hline \hline tropical storms & $375(212)$ & $247(133)$ & $128(79)$ \\
\hline minor hurricanes & $246(317)$ & $152(185)$ & $94(132)$ \\
\hline major hurricanes & $185(426)$ & $119(274)$ & $66(152)$ \\
\hline \hline all categories & $835(955)$ & $518(592)$ & $288(363)$ \\
\hline
\end{tabular}

Table 2. Optimal bandwidths in $\mathrm{km}$ for the kernel estimators for different point patterns, determined by likelihood cross validation. Point patterns: genesis - points of cyclone genesis, TS - landfall points of tropical storms, minor - landfall points of minor hurricanes, major - landfall points of major hurricanes, all - landfall points of tropical cyclones of all categories combined

\begin{tabular}{cccc}
\hline point pattern & 1903-2005 & warm phase & cold phase \\
\hline \hline genesis & 165 & 197 & 254 \\
\hline TS & 40 & 48 & 72 \\
\hline minor & 42 & 58 & 63 \\
\hline major & 39 & 44 & 49 \\
\hline all & 31 & 37 & 42 \\
\hline
\end{tabular}

Table 3. Percentages of areas with significant differences $(\alpha=$ 0.05 ) in the standardized estimated density underlying the points of cyclone genesis in the warm and the cold phase

\begin{tabular}{cccc}
\hline estimator & $\begin{array}{c}\text { higher density } \\
\text { in cold phase }\end{array}$ & $\begin{array}{c}\text { higher density } \\
\text { in warm phase }\end{array}$ & $\begin{array}{c}\text { total significant } \\
\text { differences }\end{array}$ \\
\hline \hline GNN & $30.2 \%$ & $7.5 \%$ & $37.7 \%$ \\
\hline kernel & $35.7 \%$ & $7.3 \%$ & $42.9 \%$ \\
\hline
\end{tabular}

Table 4. Results of the Monte Carlo tests of the hypotheses (6) of overall equal density fields underlying the points of cyclone genesis during the warm and the cold phase

\begin{tabular}{ccc}
\hline estimator & $p$-value & $\begin{array}{c}H_{0} \text { rejected } \\
\text { at level } \alpha=0.05\end{array}$ \\
\hline \hline GNN & 0.004 & yes \\
\hline kernel & 0.010 & yes \\
\hline
\end{tabular}

Table 5. Percentages of areas with significant differences $(\alpha=$ 0.05 ) in the standardized estimated density underlying the points of cyclone landfall in the warm and the cold phase; GNN estimator. Categories: TS - tropical storms, minor - minor hurricanes, major - major hurricanes, all - all categories combined

\begin{tabular}{cccc}
\hline category & $\begin{array}{c}\text { higher density } \\
\text { in cold phase }\end{array}$ & $\begin{array}{c}\text { higher density } \\
\text { in warm phase }\end{array}$ & $\begin{array}{c}\text { total significant } \\
\text { differences }\end{array}$ \\
\hline \hline TS & $34.2 \%$ & $21.4 \%$ & $55.6 \%$ \\
\hline minor & $18.2 \%$ & $5.3 \%$ & $23.5 \%$ \\
\hline major & $26.2 \%$ & $3.7 \%$ & $29.9 \%$ \\
\hline \hline all & $35.1 \%$ & $16.5 \%$ & $51.6 \%$ \\
\hline
\end{tabular}

Table 6. Percentages of areas with significant differences $(\alpha=$ 0.05 ) in the standardized estimated density underlying the points of cyclone landfall in the warm and the cold phase; kernel estimator of type (1). Categories: TS - tropical storms, minor - minor hurricanes, major - major hurricanes, all - all categories combined

\begin{tabular}{cccc}
\hline category & $\begin{array}{c}\text { higher density } \\
\text { in cold phase }\end{array}$ & $\begin{array}{c}\text { higher density } \\
\text { in warm phase }\end{array}$ & $\begin{array}{c}\text { total significant } \\
\text { differences }\end{array}$ \\
\hline \hline TS & $22.4 \%$ & $12.2 \%$ & $34.6 \%$ \\
\hline minor & $15.7 \%$ & $34.5 \%$ & $50.1 \%$ \\
\hline major & $12.7 \%$ & $49.5 \%$ & $62.2 \%$ \\
\hline \hline all & $16.9 \%$ & $22.5 \%$ & $48.3 \%$ \\
\hline
\end{tabular}

Table 7. Results of the Monte Carlo tests of the hypotheses (6) of overall equal density fields underlying the points of cyclone landfall during the warm and the cold phase. Categories at landfall: TS - tropical storms, minor - minor hurricanes, major - major hurricanes, all - all categories combined

\begin{tabular}{cccc}
\hline $\begin{array}{c}\text { category at } \\
\text { landfall }\end{array}$ & estimator & $p$-value & $\begin{array}{c}H_{0} \text { rejected } \\
\text { at level } \alpha=0.05\end{array}$ \\
\hline \hline TS & GNN & 0.095 & no \\
\hline TS & kernel & 0.488 & no \\
\hline minor & GNN & 0.170 & no \\
\hline minor & kernel & 0.477 & no \\
\hline major & GNN & 0.395 & no \\
\hline major & kernel & 0.014 & yes \\
\hline all & GNN & 0.524 & no \\
\hline all & kernel & 0.532 & no \\
\hline
\end{tabular}




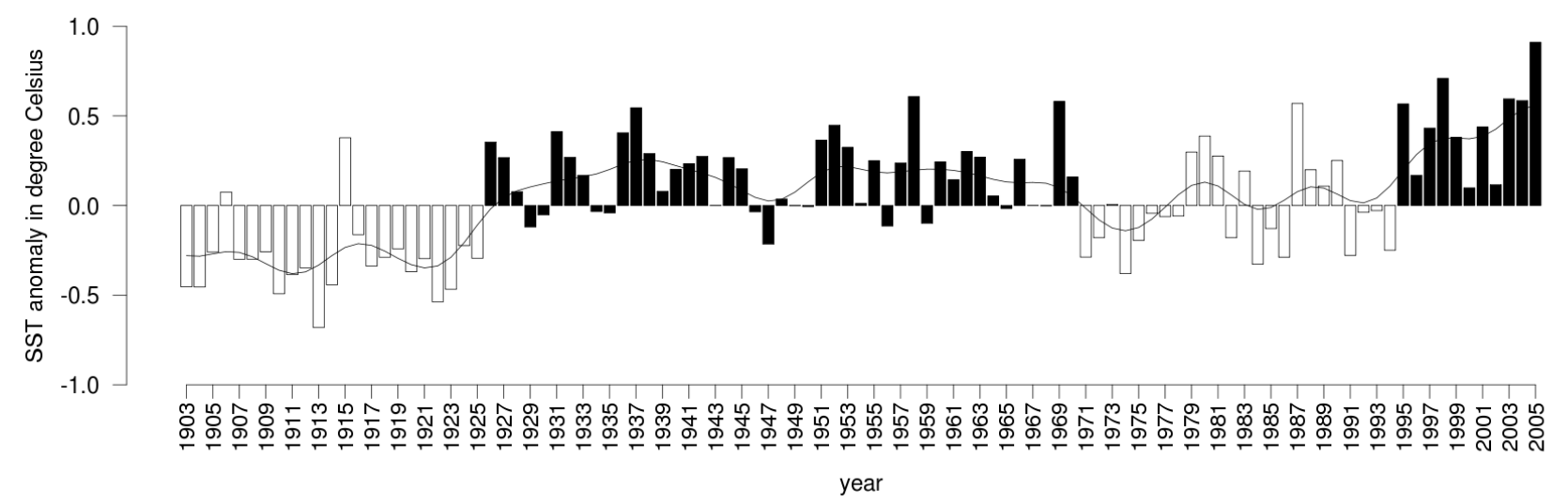

Figure 2. North Atlantic SST data; columns: deviations from the mean (in degree Celsius); solid line: smoothed mean of deviations; years marked white: cold phase; years marked black: warm phase

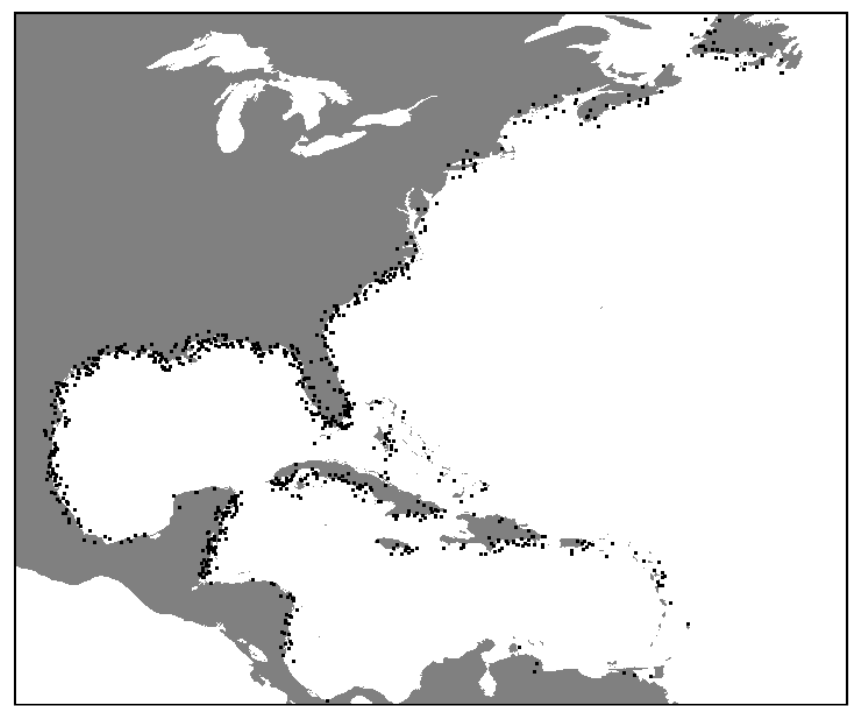

Figure 1. Investigated points of tropical cyclone landfall along the North Atlantic ocean basin 


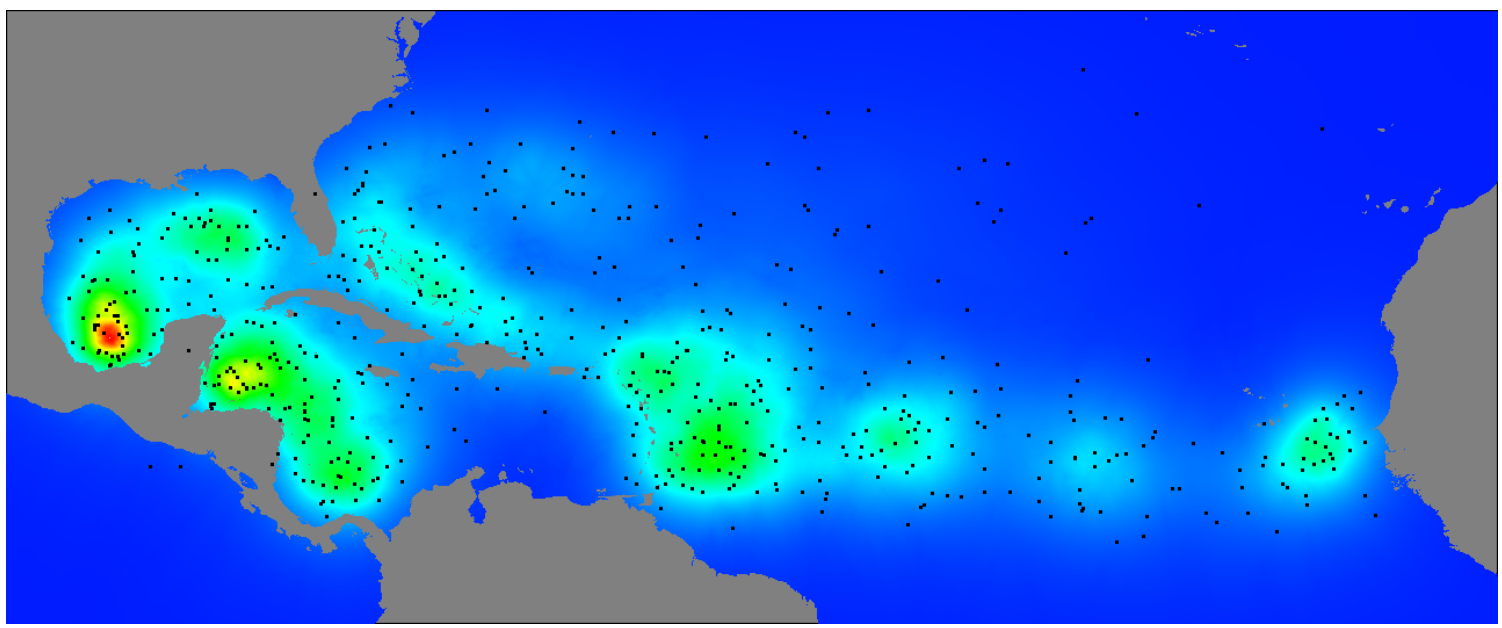

Figure 3. Standardized GNN density estimator for points of cyclone genesis from the warm phase. Historical cyclone genesis sites are marked by black points.

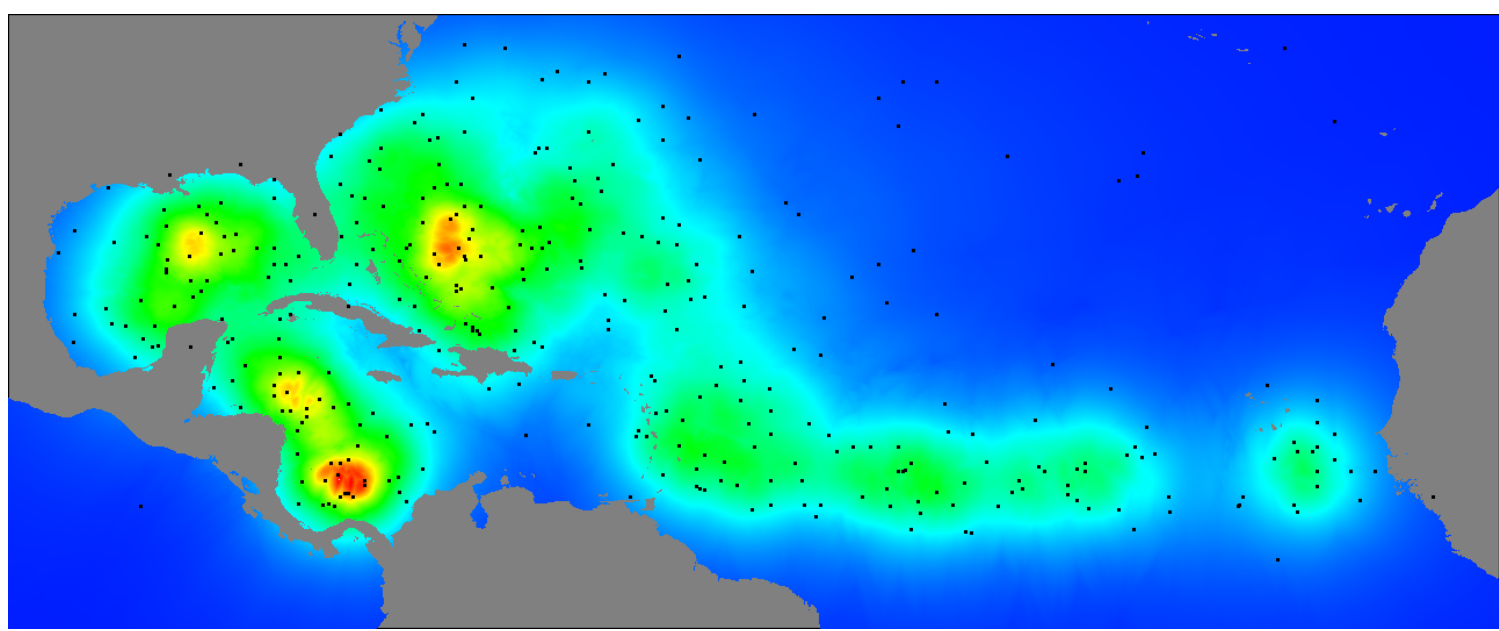

Figure 4. Standardized GNN density estimator for points of cyclone genesis from the cold phase. Historical cyclone genesis sites are marked by black points.

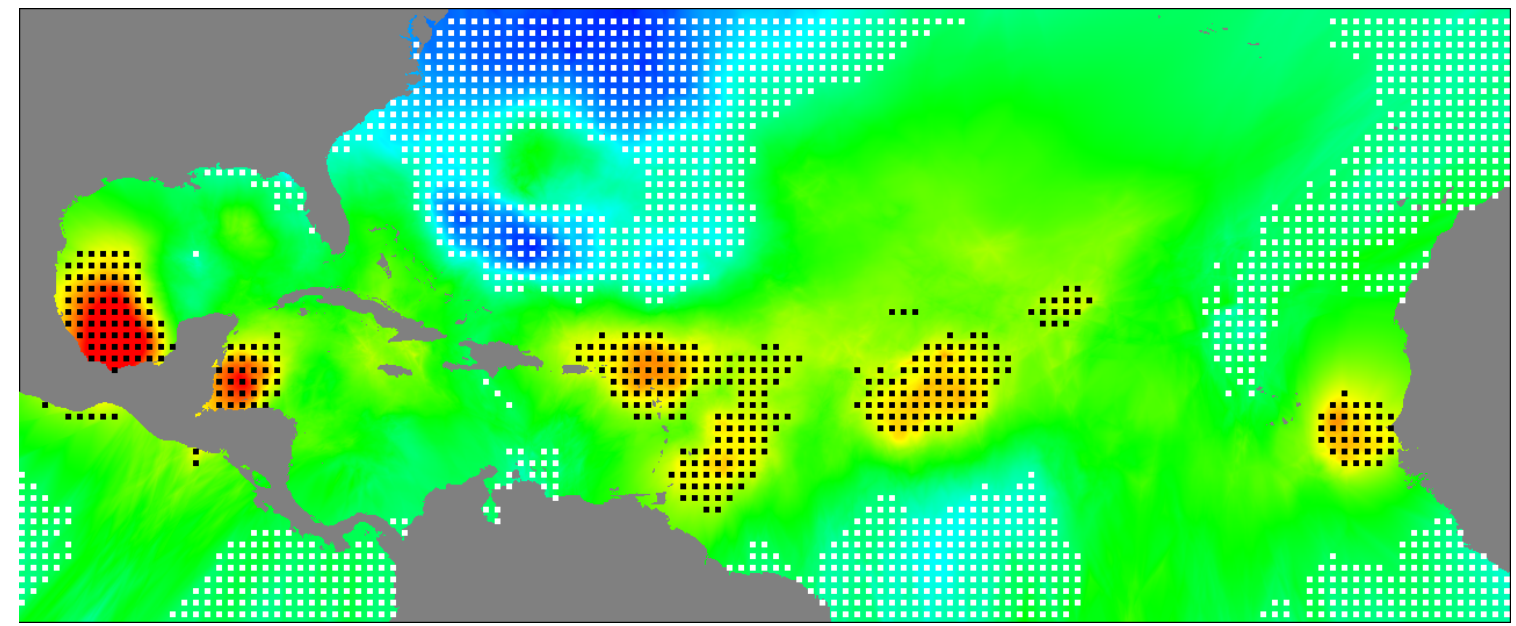

Figure 5. Log-ratio of standardized GNN density estimators for points of cyclone genesis from the warm phase and the cold phase and areas of statistically significant differences at level of significance $\alpha=0.05$. Areas overlaid with a black grid have a significantly higher standardized density during the warm phase, areas overlaid with a white grid have a significantly higher standardized density during the cold phase. 


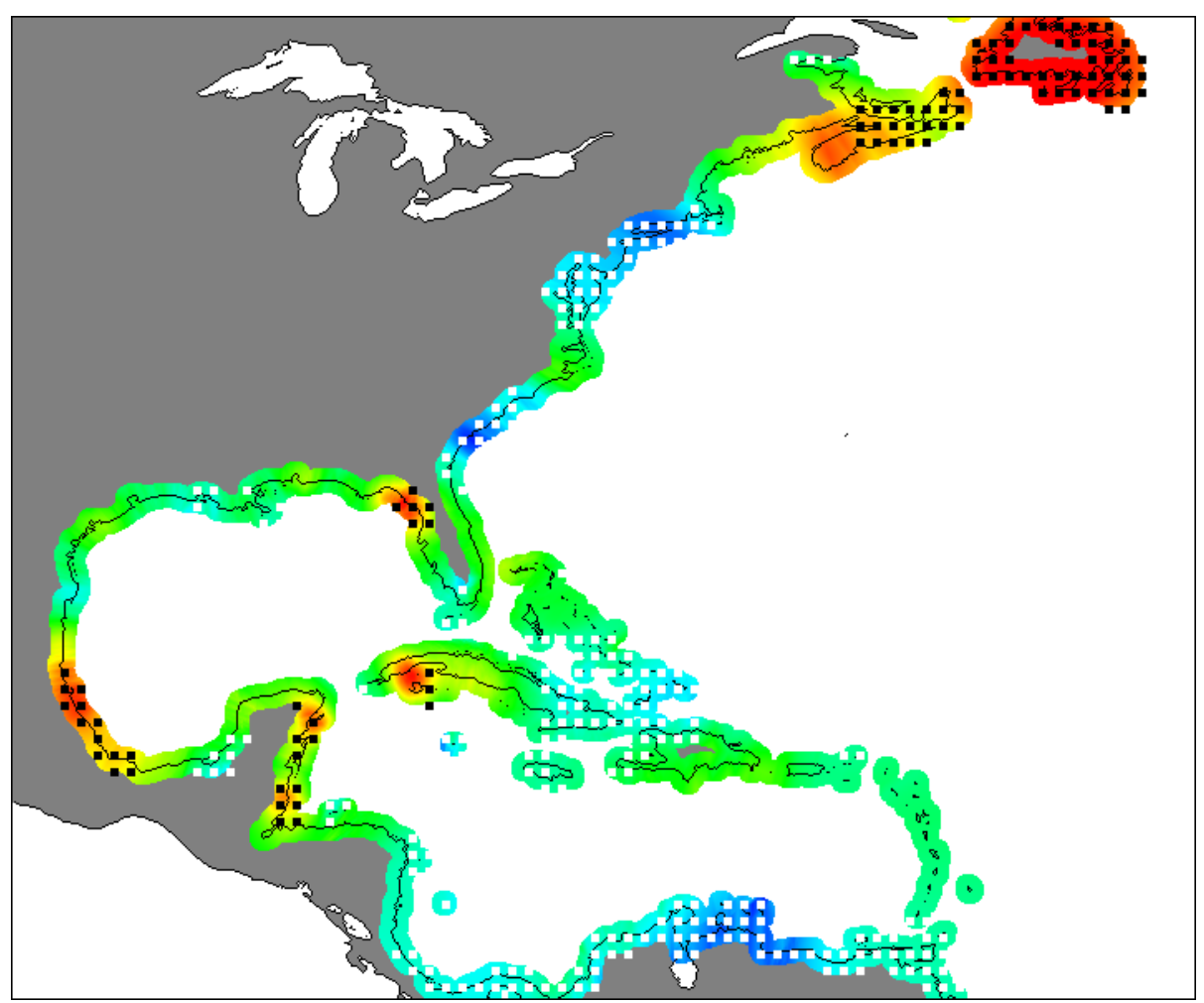

Figure 6. Log-ratio of standardized GNN density estimators for points of cyclone landfall (all categories) from the warm phase and the cold phase and areas of statistically significant differences at level of significance $\alpha=0.05$. Meaning of black and white grids as in Figure 5 .

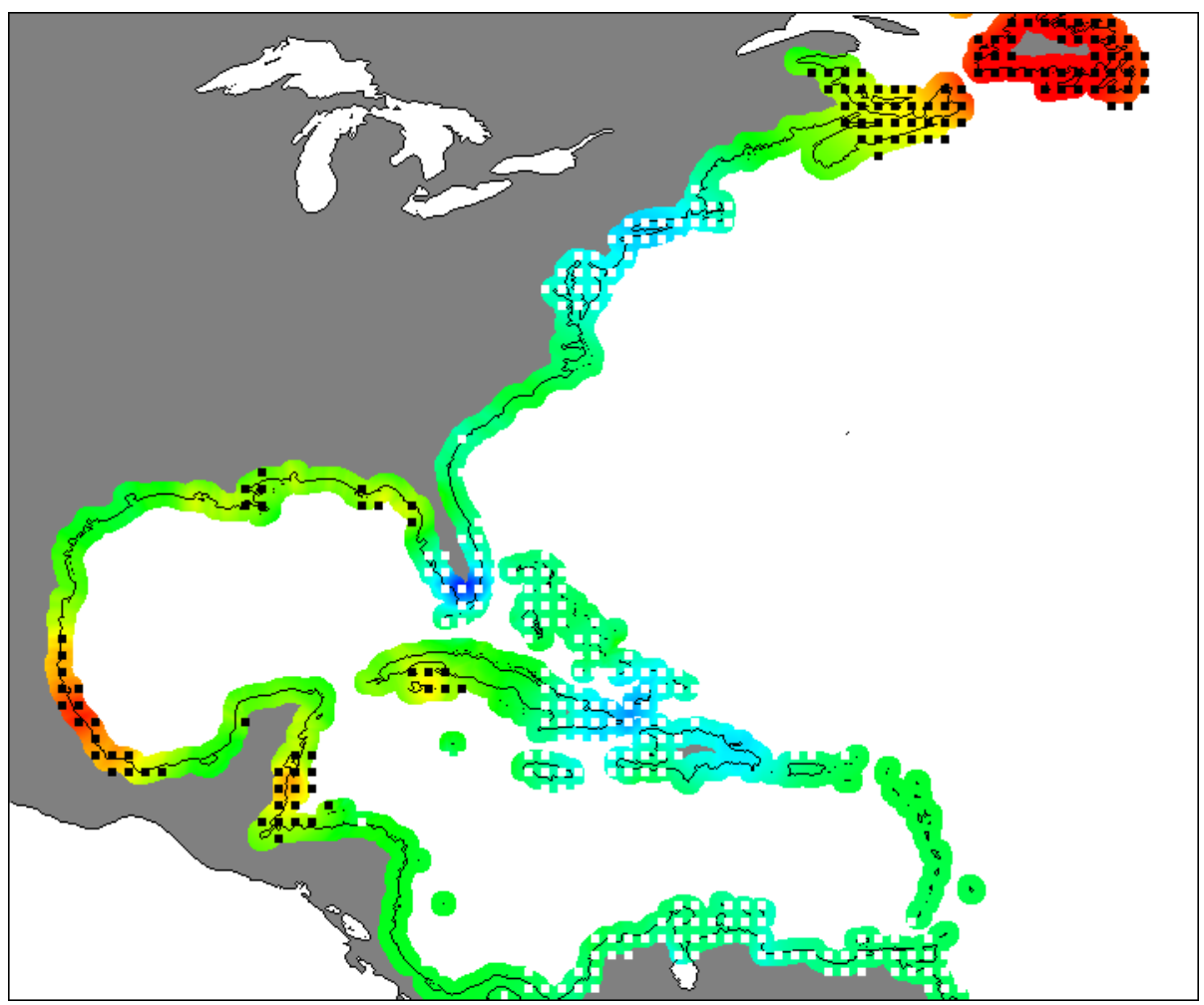

Figure 7. Log-ratio of standardized GNN density estimators for points of cyclone landfall (tropical storms) from the warm phase and the cold phase and areas of statistically significant differences at level of significance $\alpha=0.05$. Meaning of black and white grids as in Figure 5 . 


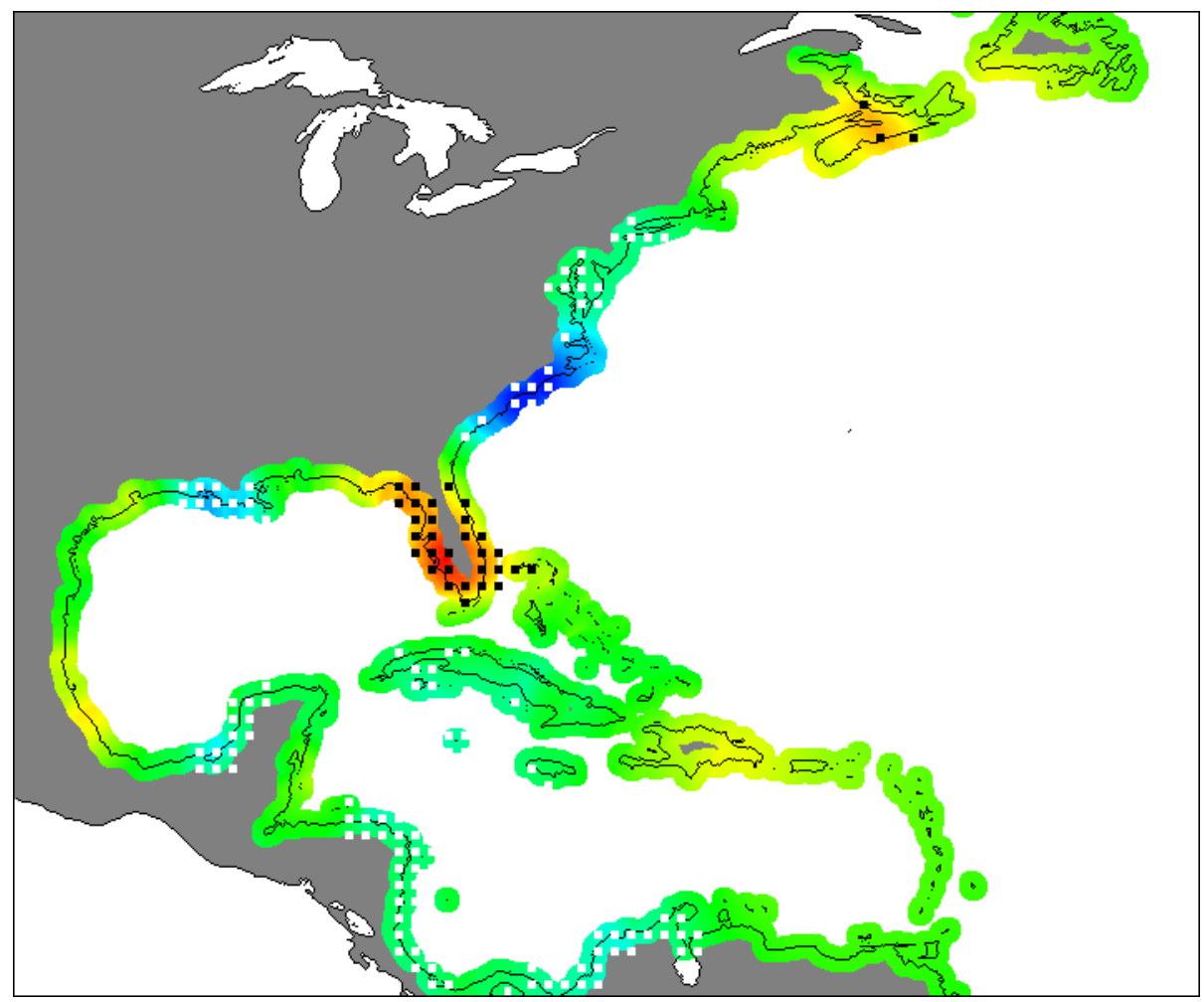

Figure 8. Log-ratio of standardized GNN density estimators for points of cyclone landfall (minor hurricanes) from the warm phase and the cold phase and areas of statistically significant differences at level of significance $\alpha=0.05$. Meaning of black and white grids as in Figure 5 .

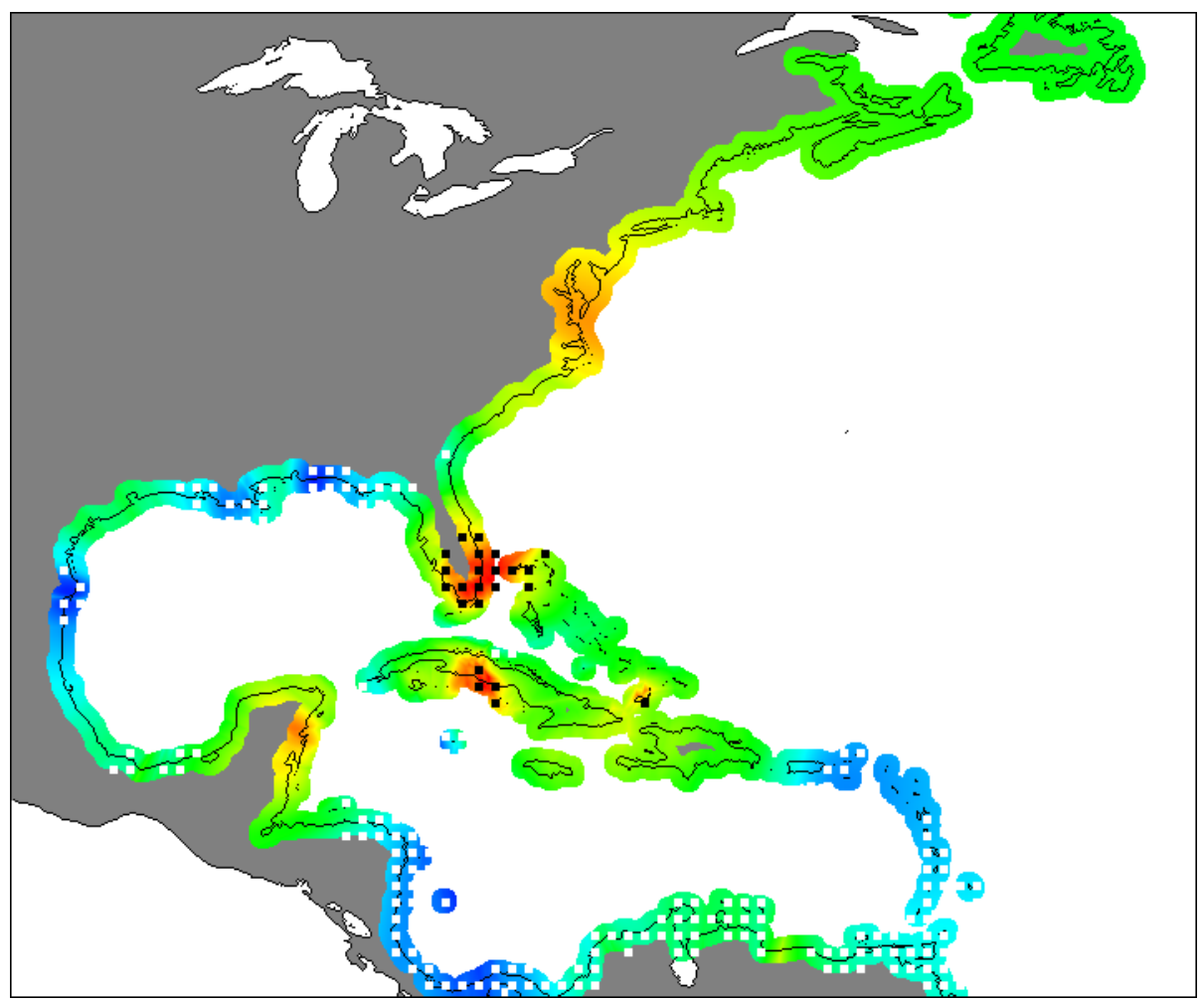

Figure 9. Log-ratio of standardized GNN density estimators for points of cyclone landfall (major hurricanes) from the warm phase and the cold phase and areas of statistically significant differences at level of significance $\alpha=0.05$. Meaning of black and white grids as in Figure 5. 


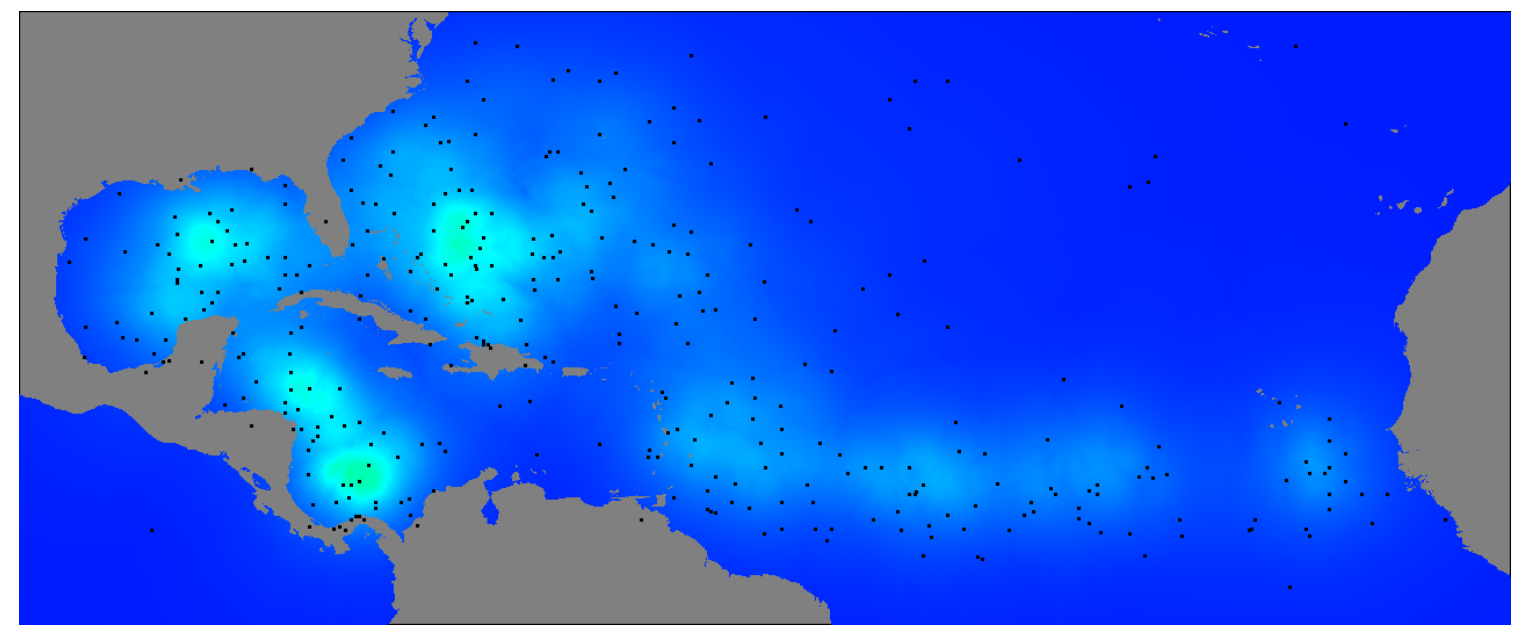

Figure 10. Non-standardized GNN density estimator for points of cyclone genesis from the cold phase. Historical cyclone genesis sites are marked by black points. Colour scale identical to that of Figure 3.

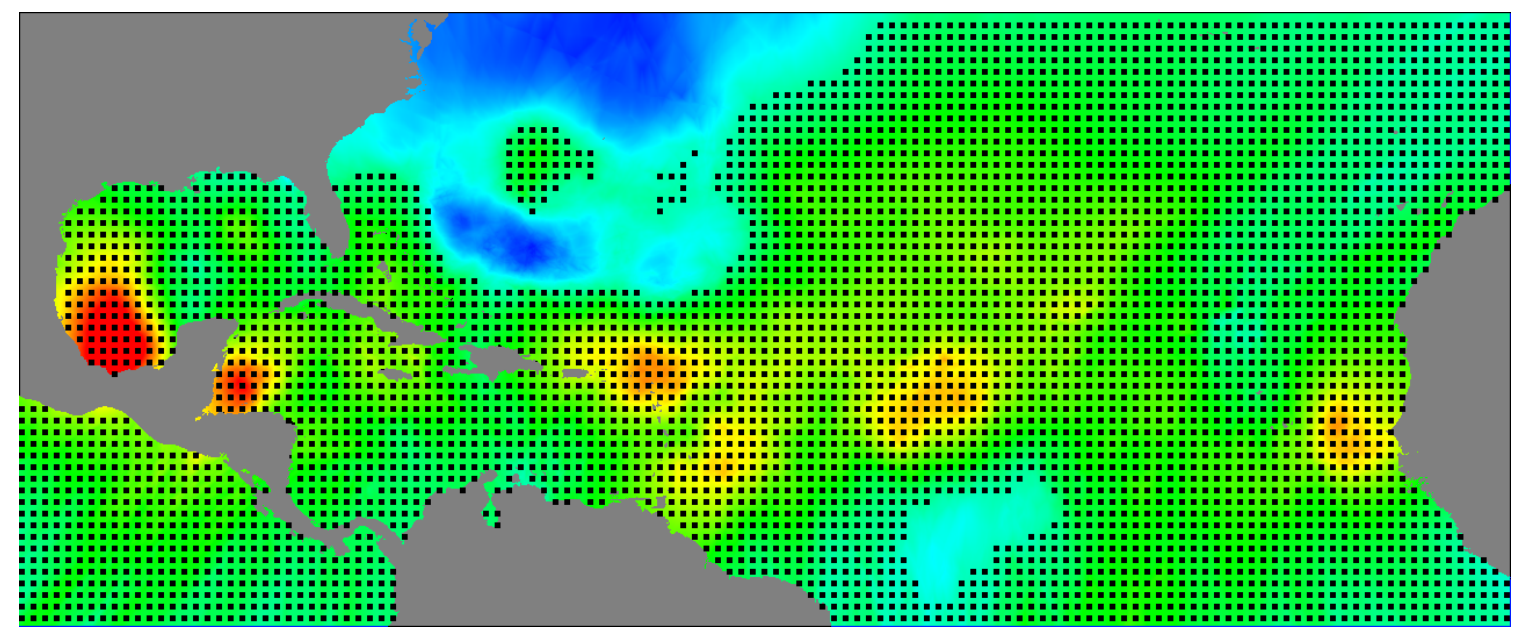

Figure 11. Log-ratio of non-standardized GNN density estimators for points of cyclone genesis from the warm phase and the cold phase and areas of statistically significant differences at level of significance $\alpha=0.05$. Areas overlaid with a black grid have a significantly higher non-standardized density during the warm phase. Note that the colours are the same as in Figure 5, although the numerical values are different, since the values depicted in Figure 5 can be obtained from those of this figure by multiplication with a constant scaling factor, i. e. the ratio of the standardization constants of the two densities. 


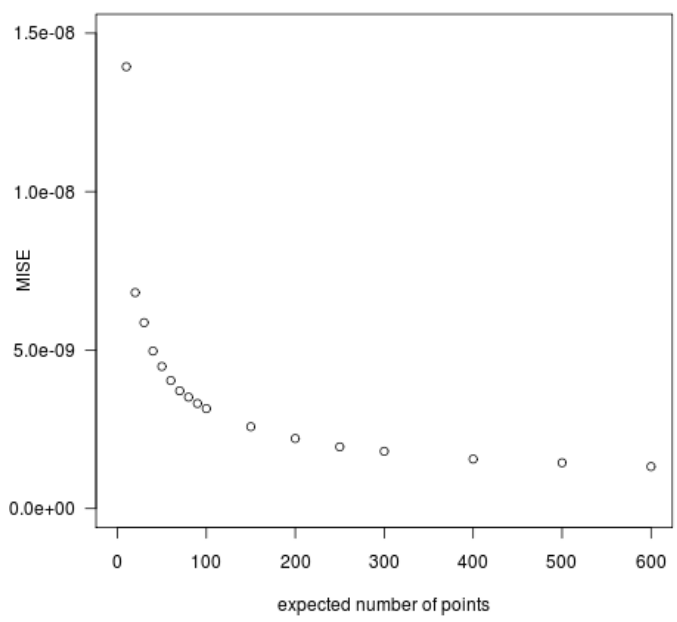

Figure 12. Results of a simulation study assessing the mean integrated squared error (MISE) of a density estimate on the observation window $W$ used for the points of cyclone landfall. Horizontal axis: expected number of points in the point pattern; vertical axis: (approximate) MISE of the kernel density estimate. 